PROCEEDINGS OF THE

AMERICAN MATHEMATICAL SOCIETY

Volume 130, Number 2, Pages 591-594

S 0002-9939(01)06073-7

Article electronically published on July 25, 2001

\title{
ON PERFECTLY MEAGER SETS IN THE TRANSITIVE SENSE
}

\author{
TOMASZ WEISS
}

(Communicated by Carl G. Jockush, Jr.)

\begin{abstract}
We prove that assuming $\mathfrak{c} \leq \aleph_{2}$ one can always find a perfectly meager set, which is not perfectly meager in the transitive sense.
\end{abstract}

In the paper [NSW] it was shown that the algebraic sum of a strongly meager set and a set of strong measure zero has to be an $s_{0}$-set. Going over the proof of this fact one can easily see that it is based on the following property of strongly meager sets.

Definition 1. A set $X \subseteq 2^{\omega}$ is said to be an $A F C^{\prime}$ set (or perfectly meager in the transitive sense) if for any perfect $P \subseteq 2^{\omega}$, there is $F$, an $F_{\sigma}$ set containing $X$, such that for each $t \in 2^{\omega},(F+t) \cap P$ is meager in the relative topology of $P$.

In $[\mathrm{N}]$ and $[\mathrm{NSW}]$ it was proven that many other well-known special subsets of the reals like $\gamma$-sets or $w Q N$-sets are perfectly meager in the transitive sense. The results appearing in those papers show that one can deduce from $Z F C$ alone the existence of an uncountable $A F C^{\prime}$ set. On the other hand, it is relatively consistent with $Z F C$ that not every perfectly meager set has to be an $A F C^{\prime}$ set (see also NW1]). Thus, it was natural to ask if the class $A F C$ of perfectly meager sets can be equal to the class $A F C^{\prime}$.

In this paper we prove that the answer is negative if we let $\mathfrak{c} \leq \aleph_{2}$. We obtain it by showing that if one assumes $\mathfrak{c} \leq \aleph_{2}$, then $A F C^{\prime}$ is strictly included in $\overline{A F C}$, where $\overline{A F C}$ denotes some subclass of $A F C$ defined below. Most of the arguments needed to show the latter fact can be found in $[R]$ and [NSW]. Throughout the paper a set of real numbers is identified with a subset of the Cantor set $2^{\omega}$. By "+" we denote the usual modulo 2 coordinatewise addition in $2^{\omega}$ and for $A, B \subseteq 2^{\omega}$, $A+B=\{a+b: a \in A, b \in B\}$. We assume that the reader is familiar with standard definitions and terminology of special sets of real numbers.

Definition 2. A set $X \subseteq 2^{\omega}$ belongs to the class $\overline{A F C}$ (of universally meager sets) iff for every $Y \subseteq 2^{\omega}$ for which there exists a one-to-one Borel measurable function $f: Y \rightarrow X$, we have that $Y \in M G R$ (meager sets).

Theorem 1 (Folklore). $\overline{A F C} \subseteq A F C$.

Proof. Suppose that $X \in \overline{A F C}$. Let $P$ be a given perfect set and let $h: P \stackrel{\text { onto }}{\longrightarrow} 2^{\omega}$ be a homeomorphism. Clearly, if $X \cap P$ is non-meager in the relative topology of $P$, then $h[X \cap P]$ is non-meager in $2^{\omega}$, but this contradicts the fact that $X \in \overline{A F C}$.

Received by the editors December 13, 1999 and, in revised form, June 27, 2000.

2000 Mathematics Subject Classification. Primary 03E15, 03E20, 28 E15. 
Theorem 2. $A F C^{\prime} \subseteq \overline{A F C}$.

Proof. See Theorem 2 in [NW1.

Theorem 3. Suppose that there is a universally meager set of cardinality $\mathfrak{c}$. Then $\overline{A F C} \neq A F C^{\prime}$.

Proof. Let $C, D$ be disjoint, perfect subsets of $2^{\omega}$ with the following property (see [NW1]):

$$
(C+C) \cap(D+D)=\{0\} .
$$

Choose $X \in \overline{A F C}$ with $|X|=\mathfrak{c}$. Let $f: 2^{\omega} \stackrel{\text { onto }}{\longrightarrow} C$ be a homeomorphism and put $Y=f[X]$. Obviously, $Y$ has to be an $\overline{A F C}$ set. From now on we follow I. Reclaw's argument from $\left[\mathrm{R}\right.$. Let $\left\{B_{y}\right\}_{y \in Y}$ be an enumeration of all $F_{\sigma}$ subsets of $2^{\omega}$. For $y \in Y$, take any $z_{y} \in D+y$ with $z_{y} \notin B_{y}$. If this is impossible, let $z_{y}$ be any element of $D+y$. It is not hard to see (use $(+))$ that $Z=\left\{z_{y}: y \in Y\right\}$ belongs to $\overline{A F C}$ as a continuous one-to-one inverse image of $Y$. By the construction, if $Z \subseteq B_{y}$ for some $y \in Y$, then $D \subseteq B_{y}+y$. Thus $Z$ is not an $A F C^{\prime}$ set.

Assume that $G$ is a family of subsets of $2^{\omega}$. For $X \subseteq 2^{\omega}$, we will say that $G$ is an $\omega$-cover of $X$ iff for any finite set $X^{\prime} \subseteq X$ there exists $g \in G$, so that $X^{\prime} \subseteq g$. Let us also recall that by $\mathfrak{b}$ we denote the $\min \{|B|: B$ is an unbounded subset of $\omega^{\omega}$ in the quasi-order $\left.\leq^{*}\right\}$.

Lemma 1. Suppose that $\mathfrak{b}=\aleph_{1}$. Then there is $X \subseteq 2^{\omega},|X|=\aleph_{1}$, such that for any sequence $\left\{G_{n}\right\}_{n \in \omega}$ of open $\omega$-covers of $X$, one can find $A \in[\omega]^{\omega},\left\{g_{n}\right\}_{n \in A}$ with every $g_{n} \in G_{n}$ and a countable $Y \subseteq X$ satisfying $X \backslash Y \subseteq \bigcup_{k \in \omega} \bigcap_{n \geq k} g_{n}$.

Proof. See Theorem 5.1 in [JMSS].

We call any set with the latter property an $S_{1}^{*}(\Omega, \Gamma)$ set and if we assume in the definition of an $X \in S_{1}^{*}(\Omega, \Gamma)$ that $A=\omega$ and $Y=\emptyset$, then $X$ is said to be a $\gamma$-set.

In the next lemma we show that any $X \in S_{1}^{*}(\Omega, \Gamma)$ is an add (meager) - small set, that is, for every sequence $\left\{G_{n}\right\}_{n \in \omega}$ of open covers of $X$, there exist $\left\{g_{n}\right\}_{n \in \omega}$ with every $g_{n} \in G_{n}$ and an increasing function $f \in \omega^{\omega}$ such that each $x \in X$ belongs to all but finitely many sets of the form $\left.\bigcup_{f(n) \leq j<f(n+1)} g_{j}(\overline{\mathrm{NSW}}]\right)$.

Lemma 2. $S_{1}^{*}(\Omega, \Gamma) \subseteq$ add (meager) - small sets.

Proof. Suppose $X \in S_{1}^{*}(\Omega, \Gamma)$ and let $\left\{G_{n}\right\}_{n \in \omega}$ be a sequence of open covers of $X$. Assume that $\left\{A_{n}\right\}_{n \in \omega}$ is an infinite partition of $\omega$ into infinite subsets. For $n \in \omega$, we define an $\omega$-cover of $X$ in the following way:

$$
F_{n}=\left\{g_{k_{1}} \cup \ldots \cup g_{k_{r}}: k_{i} \in A_{n}, g_{k_{i}} \in G_{k_{i}} \text { and } k_{i}<k_{i+1} \quad \text { for } \quad 1 \leq i \leq r\right\} .
$$

Suppose that

$$
X \backslash Y \subseteq \bigcup_{k \in \omega} \bigcap_{n \geq k} g_{n}^{\prime}
$$

where $g_{n}^{\prime} \in F_{n}$ for $n \in A, A \in[\omega]^{\omega}$ and $Y$ is a countable subset of $X$. Notice that by taking an appropriate subsequence we may assume that for every $n \in A$ :

(1) if $g_{n}^{\prime}=g_{k_{1}} \cup \ldots \cup g_{k_{r}}$ and $g_{n+1}^{\prime}=g_{m_{1}} \cup \ldots \cup g_{m_{t}}$, then $k_{r}<m_{1}$,

(2) there is $h_{n}=g_{l_{1}} \cup \ldots \cup g_{l_{s}}$ with $k_{r}<l_{1}$ and $l_{s}<m_{1}$ such that $\left\{y_{i}\right\}_{0 \leq i \leq n} \subseteq h_{n}$, where $\left\{y_{i}\right\}_{i \in \omega}$ is an enumeration of a set $Y$. 
Clearly, if we put $g_{n}^{\prime \prime}=g_{n}^{\prime} \cup h_{n}$, then

$$
X \subseteq \bigcup_{k \in \omega} \bigcap_{n \geq k} g_{n}^{\prime \prime}
$$

Lemma 3. If $X$ is an add (meager) - small set and $Y \in A F C^{\prime}$, then $X+Y \in$ $A F C^{\prime}$.

Proof. This is Theorem 24 in [NSW.

Theorem 4. Assume that $\mathfrak{c} \leq \aleph_{2}$. Then $\overline{A F C} \neq A F C^{\prime}$.

Proof. Let us consider two cases:

1. Suppose that $\mathfrak{b}=\aleph_{2}$. Then there exists a set

$$
X=\left\{f_{\alpha}: \alpha \in \aleph_{2}\right\} \subseteq \omega^{\omega \uparrow} \subseteq 2^{\omega}
$$

such that

(a) $f_{\alpha}<^{*} f_{\beta}$ for $\alpha<\beta$,

(b) $X$ is unbounded in the quasi - order $\leq^{*}$.

It is easy to show (see $\mathrm{vD}$ ) that $X$ is universally meager. Hence, by Theorem 3 , $\overline{A F C} \neq A F C^{\prime}$.

2. Let $\mathfrak{b}=\aleph_{1}$. Suppose that every $X \subseteq 2^{\omega}$ of cardinality $\aleph_{1}$ is meager. This implies (see Theorem 1 in $[\mathrm{G}]$ ) that there exists an $\overline{A F C}$ set of cardinality $\mathfrak{c}$. Thus, by Theorem $3, \overline{A F C} \neq A F C^{\prime}$. So, assume that there is a non - meager set $X$ with $|X|=\aleph_{1}$. Let $C, D$ be disjoint, perfect subsets of $2^{\omega}$ that satisfy condition (+) from the proof of Theorem 3. Suppose that $f: 2^{\omega} \stackrel{\text { onto }}{\longrightarrow} D$ is a homeomorphism. Put $Y=f[X]$. Notice that $Y \notin \overline{A F C}$. Let $Z \in S_{1}^{*}(\Omega, \Gamma)$ and $|Z|=\aleph_{1}$. We may suppose without loss of generality that $Z \subseteq C$. Define $Z^{\prime}=\left\{z+y_{z}: z \in Z\right\}$, where $\left\{y_{z}\right\}_{z \in Z}$ is an enumeration of $Y$. We have that $Z+Z^{\prime} \supseteq Y$, thus by Lemma 3, $Z^{\prime} \in \overline{A F C} \backslash A F C^{\prime}$.

Applying Theorem 1, we immediately get the main result:

Theorem 5. Let $\mathfrak{c} \leq \aleph_{2}$. Then $A F C \neq A F C^{\prime}$.

To conclude the paper let us mention that by the above argument we obtain a very simple proof of the following theorem due to A. Nowik, which gives a negative answer to M. Scheeper's question (see problem 3 in [S] and [NW2] for more details).

Theorem 6 (Nowik). It is consistent with $Z F C$ that there are a strongly measure zero set $X$ and a perfectly meager set $Y$ such that $X+Y$ is not an $s_{0}$-set.

Proof. Assume that $\mathfrak{c}=\aleph_{1}$ holds. It is well known that there exists a $\gamma$-set $X$ of cardinality $\mathfrak{c}$ (see [GM]). Clearly, $X$ is strongly measure zero. Let $C, D$ be disjoint perfect sets as in the proof of Theorem 3. Without loss of generality we may assume that $X \subseteq C$. Suppose that $\left\{y_{x}\right\}_{x \in X}$ is an enumeration of a set $D$. Define $Y=\left\{x+y_{x}: x \in X\right\}$. Obviously, $Y \in \overline{A F C}$ and we have that $X+Y \supseteq D$.

Remark. In contrast with the main theorem a parallel fact for the class $\overline{A F C}$ can not be decided by $Z F C$. This follows from a recent paper by T. Bartoszyński (see [B]) who showed that in Miller's model we have $\mathfrak{c}=\aleph_{2}$ and $A F C=\overline{A F C}$.

Finally, let us define the cardinal $\kappa$ to be equal to the least $\lambda$ such that there are no perfectly meager sets of cardinality $\lambda$. Clearly, if either $\kappa=\aleph_{2}$ or $\mathfrak{c}^{+}=\kappa$, then 
we can argue as before to show that $A F C \neq A F C^{\prime}$. It seems that for the other cases different methods have to be developed if we want to show that $A F C \neq A F C^{\prime}$. Thus we end with the following question.

Problem. Suppose that $\mathfrak{c} \geq \aleph_{3}$. Is it possible to prove on the basis of $Z F C$ that $A F C \neq A F C^{\prime} ?$

\section{REFERENCES}

[B] Bartoszyński, T.: On perfectly meager sets, preprint, 2000.

[vD] van Douwen E.: The integers and Topology, in Handbook of set-theoretic topology (K. Kunen and J.E. Vaughan, eds.), Elsevier Science Publishers, B.V., 1984, 116-167.

[G] Grzegorek, E.: Always of the first category sets, Rend. Circ. Mat. Palermo, II. Ser. Suppl. 6(1984), 139-147.

[GM] Galvin, F. and Miller, A.W.: $\gamma$-sets and other singular sets of real numbers, Topology and its Applications 17(1984), 145-155. MR 85f:54011

[JMSS] Just W., Miller A.W., Scheepers M. and Szeptycki P.: The combinatorics of open covers (II), Topology and its Applications, vol. 73 (1996), 241-266. MR 98g:03115a

[M] Miller, A.W.: Special subsets of the real line, in Handbook of set - theoretic topology (K. Kunen and J.E. Vaughan, eds), Elsevier Science Publishers B.V., 1984, 201-233. MR 86i:54037

[N] Nowik, A.: Remarks about transitive version of perfectly meager sets, Real Analysis Exchange, Volume 22(1), 1996/7, 406-412.

[NSW] Nowik, A., Scheepers, M. and Weiss, T.: The algebraic sum of sets of real numbers with strong measure zero sets, The Journal of Symbolic Logic, Volume 63, No 1, March 1998, 301-324. MR 99c:54049

[NW1] Nowik, A. and Weiss, T.: Not every Q-set is perfectly meager in the transitive sense, Proceedings of the American Mathematical Society, Volume 128, Number 10, 2000, 30173024. MR 2000m:03116

[NW2] Nowik, A. and Weiss, T.: The algebraic sum of a strong measure zero set and a perfectly meager set revisited, East-West Journal of Mathematics, Volume 2, Number 2, 2000, 191-194. CMP 2001:11

[R] Reclaw, I.: Some additive properties of special subsets of reals, Colloquium Mathematicum, Volume LXII, 1991, 221-226. MR 93b:28003

[S] Scheepers, M.: Additive properties of sets of real numbers and an infinite game, Questiones Mathematicae, 16(1993), 177-191. MR 94e:04003

WSRP, 08-110 Siedlce, Poland

E-mail address: weiss@wsrp.siedlce.pl 